\title{
ELASTODYNAMIC SINGULAR POINTS AND THEIR APPLICATIONS
}

\author{
A.D. ALAWNEH \\ Department of Mathematics \\ Jordan Universtiy \\ Amman, Jordan \\ R.P. KANWAL \\ Department of Mathematics \\ Pennsylvania State University \\ University Park, PA 16801 \\ (Received October 13, 1980)
}

ABSTRACT. Suitable singularites such as a dynamical Kelvin quadropole are defined to study the dynamical displacements set up in an infinite homogeneous and isotropic elastic medium. Approximate solutions are presented up to terms which are of higher order than those known so far.

KEY WOKDS AND PHRASES. Fundamental soxution, Doublet, quadrople, centre of rotation, displacement field, distribution of singularetes, force, spheroid.

1980 MATHEMATICS SUUJECT CLASSIFICATIUN CUDES. 73030, $46 \mathrm{~F}$.

1. INTRODUCTION.

In the recent studies in the displacement type boundary value problems the solutions have been obtained by matched asymptotic expansions [1], integral equation techniques [2] and singularity methods [3-5]. The singularity methods are based on distributing the fundamental solutions and multipoles, and centres of rotation on suitable lines, curves and surfaces $[6,7]$. By these methods it is possible to allow various dimensionless parameters inherent in the problem to vary independently. Furthermore, these methods enable us to obtain the solutions ot asymmetrical boundary value problems as well [8]. However, all the dynamical solutions presented so far are derived up to the first order of approximation. 
Our aim in this paper is to introduce the steady-state dynamic singularities and apply them to obtain the solutions for the rectilinear oscillations of inclusions embedded in an infinite homogeneous isotropic medium up to a higher order accuracy.

\section{DYNAMIC SINGULAR POINTS.}

The dynamical equations of elasticity are

$$
(\lambda+\mu) \operatorname{grad} \operatorname{div} \underset{\sim}{\mathrm{u}}+\mu \nabla^{2} \underset{\sim}{\mathrm{u}}-\rho \frac{\partial^{2} \underset{\sim}{\sim}}{\partial t^{2}}+\underset{\sim}{f}=0,
$$

where $\underset{\sim}{u}$ is the displacement vector, $\lambda$ and $\mu$ are Lame's constants of the elastic medium, $\rho$ is the density of the medium and $\underset{\sim}{f}$ is the body force per unit volume. Let us assume that $\underset{\sim}{f}=\underset{\sim}{f} e^{i \omega t}, \underset{\sim}{u}=u_{0} e^{i \omega t}$. Substituting these values in (2.1) and dropping the zero subscript, we have

$$
(\lambda+\mu) \operatorname{grad}(\operatorname{div} \underset{\sim}{\mathrm{u}})+\mu \nabla^{2} \underset{\sim}{\mathrm{u}}+\rho \omega^{2} \underset{\sim}{\mathrm{u}}+\underset{\sim}{\mathrm{f}}=0,
$$

or

$$
\mu\left[\frac{1}{1-2 v} \operatorname{grad} \operatorname{div} \underset{\sim}{\mathrm{u}}+\nabla^{2} \underset{\sim}{\mathrm{u}}+\mathrm{m}^{2} \underset{\sim}{\mathrm{u}}\right]+\underset{\sim}{\mathrm{f}}=0,
$$

where $\mathrm{m}^{2}=\rho \omega^{2} / \mu$, and $\nu$ is the Poisson ratio.

The fundamental solution $\mathrm{U}^{\mathrm{dk}}$ corresponding to the force

$$
\stackrel{\sim}{f}^{\mathrm{dk}}(\underset{\sim}{x})=4 \pi \mu \delta(\underset{\sim}{x}) \underset{\sim}{\alpha}
$$

where $\underset{\sim}{x}=\left(x_{1}, x_{2}, x_{3}\right)$ is the field point, $\delta(\underset{\sim}{x})$ is the Dirac delta function, $\underset{\sim}{\sim}$ is a constant vector and the superscripts $d$ and $k$ signify the words dynamic and Kelvin respectively, located at the origin of the coordinate system is

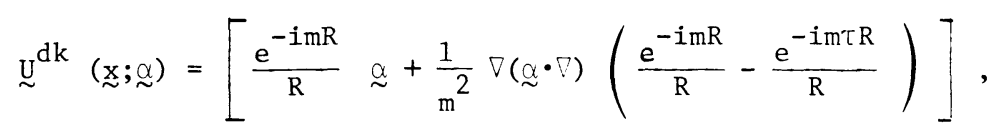

where $R=|x|$ and $\tau^{2}=(1-2 \nu) / 2(1-\nu)$. This solution will be called the dynamical Kelvin solution.

The net dynamical force $F$ experienced by a control surface $S$ enclosing the singular point is given by

$$
\begin{aligned}
\underset{\sim}{\mathrm{F}}=\int_{\mathrm{S}} \underset{\sim}{\mathrm{n}} \cdot \underset{\sim}{\mathrm{T}} \mathrm{ds}=-\int_{\mathrm{V}} \nabla \cdot \underset{\sim}{\mathrm{T}} \mathrm{dV}=\int_{\mathrm{V}} \mathrm{f}^{\mathrm{dk}} \mathrm{dV}+\mathrm{m}^{2} \mu \int_{\mathrm{V}} \overline{\mathrm{U}}^{\mathrm{dk}}(\underset{\sim}{\mathrm{x}} ; \underset{\sim}{\alpha}) \mathrm{dV} \\
=4 \pi \underset{\sim}{\alpha}+\mathrm{m}^{2} \mu \int_{\mathrm{V}} \overline{\mathrm{U}} \mathrm{dk} \underset{\underset{\sim}{\mathrm{x}}, \underset{\sim}{\alpha}) \mathrm{dV},}{ }
\end{aligned}
$$


where $\underset{\sim}{\mathrm{n}}$ is the unit outward normal to $\mathrm{S}, \underset{\sim}{\mathrm{T}}$ is the stress tensor and $\mathrm{V}$ is the cont.rol volume enclosed by $S$. 'Ihe net moment $\underset{\sim}{\mathrm{M}}$ is clearly zero.

In free space a derivative of (2.5) of any order in arbitrary direction is also a solution of (2.3). The corresponding forcing function is the derivative of $\stackrel{f}{d k}^{\mathrm{dk}}$ the same order in the same direction. These derivatives can be obtained easily by expanding $\underset{\sim}{\omega} \mathrm{dk}(\underset{\sim}{\mathrm{x}} \beta ; \alpha)$, where $\beta$ is a given vector, in Taylor series about $\underset{\sim}{\mathrm{x}}$. This expansion is

$$
{\underset{\sim}{\mathrm{U}}}^{\mathrm{dk}}(\underset{\sim}{\mathrm{x}-\beta} ; \underset{\sim}{\alpha}) \simeq{\underset{\sim}{\mathrm{U}}}^{\mathrm{dk}}(\underset{\sim}{\mathrm{x}}, \underset{\sim}{\alpha})-(\underset{\mathcal{R}}{\beta} \nabla){\underset{\sim}{\mathrm{U}}}^{\mathrm{dk}}(\underset{\sim}{\mathrm{x}} ; \underset{\sim}{\alpha})+\frac{1}{2}(\underline{\beta} \cdot \nabla)^{2}{\underset{\sim}{\mathrm{U}}}^{\mathrm{dk}}(\underset{\sim}{\mathrm{x}} ; \underset{\sim}{\alpha})+\ldots .
$$

The interpretation of various terms on the right hand side of (2.7) is as follows. The first term is the dynamical Kelvin solution discussed above, the second term represents the dynamical Kelvin doublet characterized by the vectors $\underset{\sim}{ }$ and $\beta$, the third term gives the corresponding quadrople and so on. The dynamica1 Kelvin doublet can be written as

$$
\begin{aligned}
& {\underset{\sim}{U}}^{\mathrm{dkd}}(\underset{\sim}{\mathrm{x}} ; \underset{\sim}{\alpha}, \beta)=-(\underline{\beta} \cdot \nabla){\underset{\sim}{\mathrm{U}}}^{\mathrm{dk}}(\underset{\sim}{\mathrm{x}} ; \underset{\sim}{\alpha)}) \\
& \quad=-\underset{\sim}{\alpha}(\underset{\sim}{\beta} \cdot \nabla) \frac{\mathrm{e}^{-\mathrm{imR}}}{\mathrm{R}}-\frac{1}{\mathrm{~m}^{2}} \nabla(\underline{\beta} \cdot \nabla)(\underset{\sim}{\alpha} \cdot \nabla)\left[\frac{\mathrm{e}^{-\mathrm{imR}}}{\mathrm{R}}-\frac{\mathrm{e}^{-\mathrm{im \tau} \mathrm{R}}}{\mathrm{R}}\right],
\end{aligned}
$$

while the corresponding forcing function is

$$
\stackrel{\sim}{f}^{\mathrm{dkd}}=-4 \pi \mu(\underline{\beta} \cdot \nabla) \delta(\underset{\sim}{\mathrm{x}}) \underset{\sim}{\alpha}=-4 \pi \mu[\underset{\alpha}{\beta} \cdot \nabla \delta(\underset{\sim}{\mathrm{x}})] \underset{\sim}{\alpha},
$$

where the superscript dkd stands for the dynamical Kelvin doublet.

From relation (2.8) it follows that a dynamical Kelvin doublet is not symmetric with respect $b$ the changing of $\underset{\sim}{\alpha}$ and $\beta$. Its symmetric and antisymmetric parts yiled another important fundamental solution. The antisymmetric part is

$$
\begin{aligned}
& \left.\frac{1}{2}\left[{\underset{\sim}{U}}^{\mathrm{dkd}}(\underset{\sim}{\mathrm{x}} ; \underset{\sim}{\alpha}, \beta)-\mathrm{U}^{\mathrm{dkd}}(\underset{\sim}{\mathrm{x}} ; \underset{\sim}{\beta}, \underset{\sim}{\alpha})\right]=\frac{1}{2}[\underset{\sim}{\alpha(\beta} \cdot \nabla) \frac{\mathrm{e}^{-i \mathrm{mR}}}{\mathrm{R}}-\underset{\sim}{\alpha(\alpha \cdot \nabla)} \frac{\mathrm{e}^{-1 \mathrm{mR}}}{\mathrm{R}}\right] \\
& =-\nabla \times \frac{(\underset{\sim}{\alpha} \beta)}{2} \frac{\mathrm{e}^{-1 \mathrm{mR}}}{\mathrm{R}} \text {. }
\end{aligned}
$$

We shall call it the dynamical center of rotation and denote it as $\underset{\sim}{\mathrm{U}}{ }^{\mathrm{dr}}$. If we set $\mathcal{L}=-(\underset{\sim}{\alpha} \times \beta) / 2$, the above relation becomes

$$
{\underset{\sim}{U}}^{\mathrm{dr}}(\underset{\sim}{x}, \mathfrak{L})=\nabla \times \mathfrak{L} \frac{\mathrm{e}^{-i m R}}{\mathrm{R}},
$$

where the superscript $d r$ stands for the dynamical center of rotation. The 
corresponding forcing function is

$$
\stackrel{\sim}{\sim}^{\mathrm{dr}}=\frac{1}{2}\left[\mathrm{f}^{\mathrm{dkd}}(\underset{\sim}{\mathrm{x}} ; \underset{\sim}{\alpha}, \beta)-\mathrm{f}^{\mathrm{dkd}}(\underset{\sim}{\mathrm{x}}, \beta, \underset{\sim}{\alpha})\right]=4 \pi \mu \nabla \times[\delta(\underset{\sim}{\mathrm{x}}) \mathfrak{\chi}] .
$$

Since $\underset{\sim}{\mathrm{U}}{ }^{\mathrm{dr}}$ has only a vector potential $\chi \mathrm{e}^{-i m R} / \mathrm{R}$ and no scalar potential, the net force $\underset{\sim}{\mathrm{F}}$ vanishes while the torque $\underset{\sim}{\mathrm{M}}$ experienced by the control volume $\mathrm{V}$ containing the singular point and bounded by $S$ is

$$
\begin{aligned}
& \underset{\sim}{M}=\int_{S} \underset{\sim}{x} \times(\underset{\sim}{(n} \cdot \underset{\sim}{T}) d s=-\int_{V}(\nabla \cdot \underset{\sim}{T}) \times \underset{\sim}{x} d V
\end{aligned}
$$

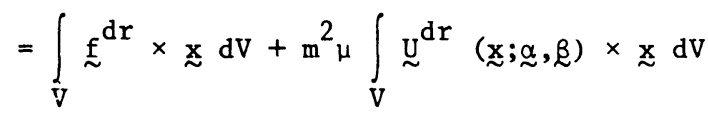

$$
\begin{aligned}
& =-8 \pi \mu \mathcal{L}+\mathrm{m}^{2} \mu \int_{\mathrm{V}}{\underset{\sim}{\mathrm{d}}}^{\mathrm{dr}}(\underset{\sim}{\mathrm{x}} ; \underset{\sim}{\alpha}, \beta) \times \underset{\sim}{\mathrm{x}} \mathrm{dV} .
\end{aligned}
$$

The symmetric part of the dynamical Kelvin doublet gives us another fundamental solution which will be called the dynamical stresslet. The corresponding displacement field and the forcing function respectively are

$$
\begin{aligned}
& {\underset{\sim}{\mathrm{U}}}^{\mathrm{dks}}(\underset{\sim}{\mathrm{x}} ; \underset{\sim}{\alpha}, \beta)=\frac{1}{2}\left[{\underset{\sim}{U}}^{\mathrm{dkd}}(\underset{\sim}{x} ; \underset{\sim}{\alpha}, \beta)+{\underset{\sim}{\mathrm{U}}}^{\mathrm{dkd}}(\underset{\sim}{x}, \alpha, \beta)\right]
\end{aligned}
$$

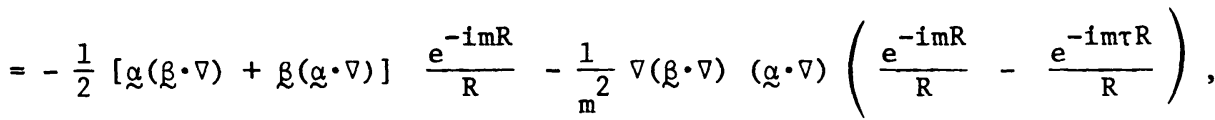

and

$$
\stackrel{\sim}{f}^{\mathrm{dks}}=-2 \pi \mu\left[\{\underline{\beta}-\nabla \delta(\underset{\alpha}{\alpha})\}_{\alpha}^{\alpha}+\{\underset{\alpha}{\alpha} \cdot \nabla \delta(\underset{x}{)})\} \underline{\alpha}\right] .
$$

This fundamental solution represents a self equilibrating system and accordingly contributes neither a net force nor a moment to the medium.

Another useful fundamental solution in the present investigation is the potential doublet which is characterized by a scalar function and is also useful in discussing vibrations in Stokes flow [7]. The displacement field due to this potential is

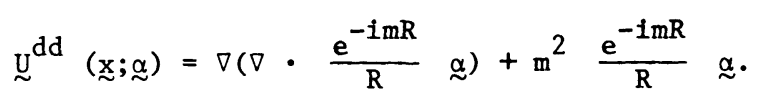


In the next section we demonstrate that these fundamental solutions are very effective in solving boundary value problems in elastodynamics. To fix the ideas we consider the case of a spheroid.

3. TRANSLATION OF A PROLATE SPHEROID.

Let the rigid spheroid $S$,

$$
\frac{x^{2}}{a^{2}}+\frac{r^{2}}{b^{2}}=1, \quad r^{2}=y^{2}+z^{2}, \quad c^{2}=\left(a^{2}-b^{2}\right)=a^{2} e^{2}
$$

where $e(0 \leq e \leq 1)$ is the eccentricity and $2 c$ is the focal length, be embedded in an isotropic and homogeneous elastic medium. It is excited by a periodic force with period $2 \pi / \omega$ acting in the direction of its axis of symmetry so that the displacement of the points on $\mathrm{S}$ is

$$
\underset{\sim}{\mathrm{U}}=\mathrm{U} \stackrel{\mathrm{e}}{\mathrm{u}}_{\mathrm{x}}
$$

where $\underset{\sim}{\hat{e}_{x}}$ is the unit vector along $x$-axis and $U$ is a constant.

Our aim is to find the displacement field in the elastic medium so that equation (2.3) and the boundary condition (3.2) as well as the far-field radiation condition are satisfied. For this purpose we apply the technique of distributing the appropriate singularities in reference [3]. In the present situation we have the following line distributions between the focii $x=-c$ and $x=c$,

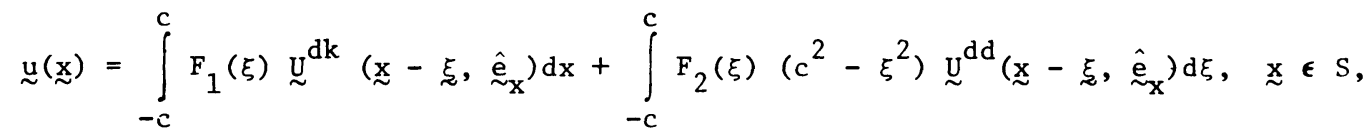

where $\xi=\xi \hat{\mathrm{e}}_{\mathbf{x}}$. The first integral in (3.3) represents the line distribution of dynamical Kelvin solutions (2.5) of variable strength $F_{1}(\xi)$ while the second integral is the line distribution of potential doublets (2.15) with the strength $\left(c^{2}-\xi^{2}\right) \mathrm{F}_{2}(\xi)$. Clearly, (3.3) satisfies the differential equation and vanishes as $|\mathbf{x}| \rightarrow \infty$.

To find $F_{1}(\xi)$ and $F_{2}(\xi)$ we apply the boundary condition (3.2) on the surface $S$ of the spheroid and get

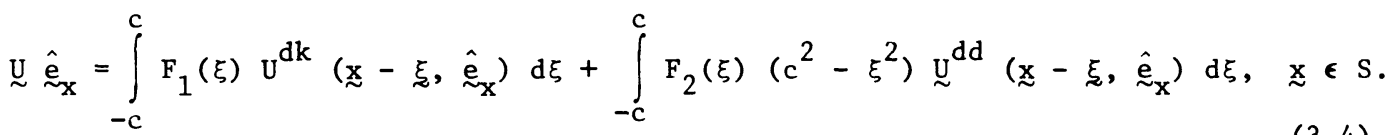


It is obvious that we cannot easily find a closed form solution of equation (3.4). Accordingly, we use a perturbation technique for small values of the parameter $\mathrm{m}$. For this purpose, we expand all the functions in (3.4) in powers of $\mathrm{m}$,

$$
\begin{aligned}
& F_{1}(\xi)=f_{11}(\xi)+m f_{12}(\xi)+m^{2} f_{13}(\xi)+0\left(m^{3}\right), \\
& \mathrm{F}_{2}(\xi)=\mathrm{f}_{21}(\xi)+\mathrm{mf}{ }_{22}(\xi)+\mathrm{m}^{2} \mathrm{f}_{23}(\xi)+0\left(\mathrm{~m}^{3}\right),
\end{aligned}
$$

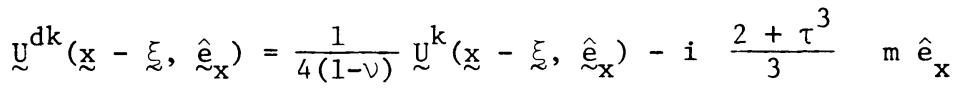

$$
\begin{aligned}
& +\left[-\frac{\left(3+\tau^{4}\right)}{8}|\underset{\sim}{x}-\xi|{\underset{\sim}{\hat{~}}}_{x}+\frac{\left(1-\tau^{4}\right)(x-\xi)(x-\xi)}{8(x-\xi)}\right] m^{2}+0\left(m^{3}\right),
\end{aligned}
$$

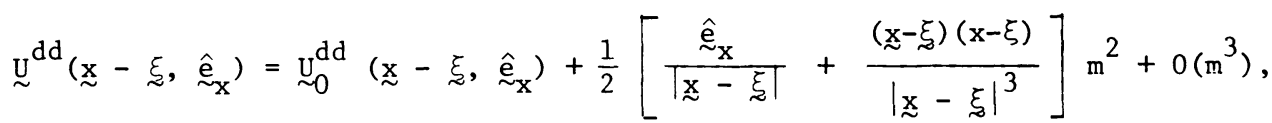

where

$$
{\underset{\sim}{\mathrm{U}}}^{\mathrm{k}}(\underset{\sim}{\mathrm{x}}, \underset{\sim}{\alpha})=\frac{(3-4 \nu) \underset{\sim}{\alpha}}{\mathrm{R}}+\frac{(\underset{\sim}{\alpha} \cdot \underset{\sim}{\mathrm{x}}) \underset{\sim}{\mathrm{x}}}{\mathrm{R}^{3}}, \quad \mathrm{U}_{0}^{\mathrm{dd}}(\underset{\sim}{\mathrm{x}} \underset{\sim}{\alpha})=-\frac{\underset{\sim}{\alpha}}{\mathrm{R}^{3}}+\frac{3(\underset{\sim}{\alpha} \cdot \underset{\sim}{\mathrm{x}}) \underset{\sim}{\mathrm{x}}}{\mathrm{R}^{5}},
$$

are the Kelvin solution and potential doublets respectively for the elastostatic field [3].

When we substitute the expansions (3.5) in (3.4) and equate the equal powers of $\mathrm{m}$ we get the following system of equations

$$
\begin{aligned}
& \frac{1}{4(1-v)} \int_{-c}^{c} f_{11}{\underset{\sim}{U}}^{k}\left(\underset{\sim}{x}-\xi, \underset{\sim}{\hat{e}_{x}}\right) d \xi+\int_{-c}^{c}\left(c^{2}-\xi^{2}\right) f_{21} \underset{\sim}{\stackrel{d}{d d}}\left(\underset{\sim}{x}-\xi, \underset{\sim}{\hat{e}_{x}}\right) d \xi=U \underset{\sim}{\hat{e}_{x}},
\end{aligned}
$$

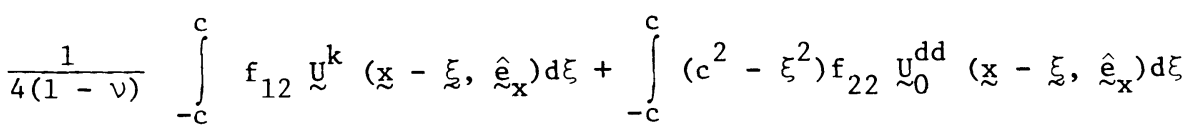

$$
\begin{aligned}
& =\hat{e}_{x} \int_{-c}^{c} \frac{i\left(2+\tau^{3}\right)}{3} f_{11} d \xi \\
& \frac{1}{4(1-\nu)} \int_{-c}^{c} f_{13}{\underset{\sim}{u}}^{k}(\underset{\sim}{x}-\xi \underset{\sim}{\underset{x}{e}}) d+\int_{-c}^{c}\left(c^{2}-\xi^{2}\right) f_{23} U_{0}^{d d}(\underset{\sim}{x}-\underset{\sim}{\xi}, \underset{\sim}{\underset{x}{e}}) d \xi \\
& =\int_{-c}^{c} f_{11}\left[\frac{3+\tau^{4}}{8} \mid \underset{\sim}{x}-\underset{d}{\mid \hat{e}_{x}}-\frac{\left(1-\tau^{4}\right)(x-\xi)(x-\xi)}{8|x-\xi|}\right] d \xi
\end{aligned}
$$




$$
-\frac{1}{2} \int_{-c}^{c}\left(c^{2}-\xi^{2}\right) f_{21}\left[\frac{\hat{e}_{x}}{|\underset{\sim}{x}-\xi|}+\frac{(x-\xi)(\underset{\sim}{x}-\xi)}{|x-\xi|^{3}}\right] d \xi+\frac{i\left(2+\tau^{3}\right)}{3}{\underset{\sim}{x}}_{-c}^{c} \int_{12}^{c} d \xi .
$$

The solutions of equations (3.6) and (3.7) are available in reference [3] so that

$$
\begin{aligned}
& \frac{1}{4(1-v)} \mathrm{f}_{11}=-\frac{2 e^{2}}{1-e^{2}} \mathrm{f}_{21}=U e^{2}\left[-2 e+\left(1+3 e^{2}-4 \gamma e^{2}\right) L\right]^{-1}, \\
& \frac{1}{4(1-v)} f_{12}=-\frac{2 e^{2}}{1-e^{2}} f_{22}=\frac{2 i\left(2+\tau^{3}\right)}{3} f_{11} a e^{3}\left[-2 e+\left(1+3 e^{2}-4 \gamma e^{2}\right) L\right]^{-1},
\end{aligned}
$$

where $L=\ln [(1+e) /(1-e)]$.

Next we substitute (3.9) and (3.10) in (3.8) and obtain

$$
\begin{aligned}
& \frac{1}{4(1-v)} \int_{-c}^{c} f_{13} \stackrel{\sim}{\sim}^{k}\left(\underset{\sim}{x}-\xi \underset{\sim}{\hat{e}_{x}}\right) d \xi+\int_{-c}^{c}\left(c^{2}-\xi^{2}\right) f_{23} \underset{\sim}{\underset{\sim}{d d}}(\underset{\sim}{x}-\xi \underset{\sim}{\hat{e}}) d \xi \\
& =\left(\psi_{0}+\psi_{1} x^{2}\right)+\psi_{2} x r{\stackrel{\hat{e}_{r}}{r}},
\end{aligned}
$$

where

$$
\begin{aligned}
& \psi_{0}=\frac{a^{2} f_{11}}{4}\left[e\left(1+\tau^{4}\right)+\left(1-e^{2}\right) L\right]-a^{2} f_{21}(-2 e+L)+\frac{2 \text { iae }\left(2+\tau^{3}\right)}{3} f_{12}, \\
& \psi_{1}=\frac{f_{11}}{4}\left[e\left(1+\tau^{4}\right)-\left(1-e^{2}\right) L\right]-f_{21}\left[4 e-\left(2-e^{2}\right) L\right], \\
& \psi_{2}=-\left[\frac{1-\tau^{4}}{4} \quad f_{11} e+f_{21}(-2 e+L)\right],
\end{aligned}
$$

and $e_{r}=\left(y \underset{\sim}{\hat{e}_{y}}+z{\underset{\sim}{\hat{e}}}_{z}\right) / r$ is the unit radial vector in the yz - plane. To solve (3.11) we set

$$
f_{13}(x)=A_{0}+A_{2} x^{2}, \quad f_{23}(x)=C_{0}+C_{2} x^{2}, \quad|x| \leq c,
$$

The substitution of relations (3.13) in (3.11) and simplification yields

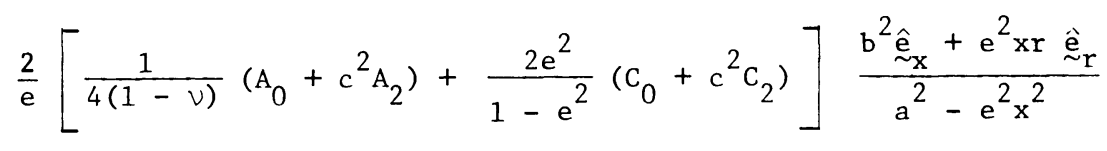

$$
\begin{aligned}
& +\left[\phi_{0} A_{0}+\phi_{1} A_{2}-2 C_{0} L+\phi_{2} C_{2}\right] \stackrel{\hat{e}}{x}^{\hat{e}_{x}} \\
& -\mathrm{x}^{2}\left(\mathrm{~A}_{2} \lambda_{1}+\mathrm{C}_{2} \lambda_{2}\right) \hat{\mathrm{e}}_{\mathrm{x}}-\left(\mathrm{A}_{2} \theta_{1}+\mathrm{C}_{2} \theta_{2}\right) \mathrm{xr} \hat{\sim}_{\mathrm{r}}=\left(\psi_{0}+\psi_{1} \mathrm{x}^{2}\right) \hat{\mathrm{e}}_{\mathrm{x}}+\psi_{2} \mathrm{xr} \hat{\mathrm{e}}_{\mathrm{r}},
\end{aligned}
$$


where

$$
\begin{gathered}
\phi_{0}=-\frac{1}{2(1-\nu) e}+\mathrm{L}, \quad \phi_{1}=a^{2}\left[\mathrm{e}-\frac{\left(1-\mathrm{e}^{2}\right)(3-2 \nu)}{4(1-v)} \mathrm{L}\right], \\
\phi_{2}=-2 \mathrm{a}^{2}\left[6 \mathrm{e}-\left(3-2 \mathrm{e}^{2}\right) \mathrm{L}\right], \quad \lambda_{1}=\frac{(14-12 \nu) e-\left(7-6 v-3 \mathrm{e}^{2}+2 v \mathrm{e}^{2}\right) \mathrm{L}}{4(1-\nu)}, \\
\lambda_{2}=-36 \mathrm{e}+2\left(9-3 \mathrm{e}^{2}\right) \mathrm{L}, \quad \theta_{1}=-\frac{2 \mathrm{e}+\mathrm{L}}{2(1-\nu)}, \theta_{2}=16 \mathrm{e}+\frac{8 \mathrm{e}}{1-\mathrm{e}^{2}}-12 \mathrm{~L} .
\end{gathered}
$$

Equation (3.14) is satisfied if we choose

$$
\begin{gathered}
\frac{1}{4(1-v)}\left(A_{0}+c^{2} A_{2}\right)=-\frac{2 e^{2}}{1-e^{2}}\left(C_{0}+c^{2} C_{2}\right), \\
\phi_{0} A_{0}+\phi_{1} A_{2}-2 L C_{0}+\phi_{2} C_{2}=\psi_{0}, \\
\theta_{1} A_{2}+\theta_{2} C_{2}=-\psi_{2}, \\
\lambda_{1} A_{2}+\lambda_{2} C_{2}=-\psi_{1} .
\end{gathered}
$$

This is a linear system of equations which has the following solution

$$
\begin{aligned}
& \mathrm{A}_{2}=\frac{\lambda_{2} \psi_{2}-\theta_{2} \psi_{1}}{\lambda_{1} \theta_{2}-\lambda_{2} \theta_{1}} \text {, } \\
& C_{2}=\frac{\theta_{1} \psi_{1}-\lambda_{1} \psi_{2}}{\lambda_{1} \theta_{1}-\lambda_{2} \theta_{1}} \\
& C_{0}=\left[\frac{8 e^{2}(1-\nu) \phi_{0}}{1-e^{2}}+2 L\right]^{-1}\left[\left\{\phi_{2}-\frac{8 a^{2}(1-\nu) e^{3}}{1-e^{2}}\right\} C_{2}-\psi_{0}+\left(\phi_{1}-c^{2} \phi_{0}\right) A_{2}\right] \text {, } \\
& A_{0}=-\frac{8 e^{2}(1-\nu)}{1-e^{2}} \quad\left(C_{0}+c^{2} C_{2}\right)-c^{2} A_{2} \cdot
\end{aligned}
$$

The net force $\underset{\sim}{\mathrm{F}}$ experienced by the spheroid can be computed by superposition of $(2.6)$;

$$
\underset{\sim}{\mathrm{F}}=4 \pi \mu \hat{\mathrm{e}}_{\mathrm{x}} \int_{-\mathrm{c}}^{\mathrm{c}}\left[\mathrm{f}_{11}(\xi)+\mathrm{mf}_{12}(\xi)+\mathrm{m}^{2} \mathrm{f}_{13}(\xi)+0\left(\mathrm{~m}^{3}\right)\right] \mathrm{d} \xi+\mu \mathrm{m}^{2} \int_{\mathrm{V}} \overline{\mathrm{u}} \mathrm{dV},
$$

where the volume integral is taken over the spheroid. When we substitute the values 
of $\mathrm{f}_{11}, \mathrm{f}_{12}, \mathrm{f}_{13}$ and $\underset{\sim}{\mathrm{u}}$ in the above formula, we have

$$
\begin{aligned}
\underset{\sim}{\mathrm{F}=} \mathrm{P}_{0}\left[1+\frac{\mathrm{P}_{0} i\left(\tau^{3}+2\right)}{12 \pi \mu \mathrm{a} U} \mathrm{am}\right]+\frac{8 \pi \mu \mathrm{ae}}{3 \mathrm{P}_{0}} & \left\{\frac{3 \mathrm{~A}_{0}}{\mathrm{a}^{2}+\mathrm{A}_{2} \mathrm{e}^{2}+\mathrm{f}_{21}\left[2-4(1-\nu) \mathrm{e}^{2}\right.}\right. \\
& \left.\left.\left.-\left(\mathrm{e}^{-1}+4 \mathrm{e}-5 v \mathrm{e}\right) \mathrm{L}\right]\right\}(\mathrm{am})^{2}\right] \mathrm{e}_{\mathrm{x}}+0(\mathrm{am})^{3},
\end{aligned}
$$

where

$$
P_{0}=32 \pi \mu a(1-\nu) U e^{3}\left[-2 e+\left(1+3 e^{2}-4 v e^{2}\right) L\right]^{-1}
$$

Relation (3.24) agrees with Kanwal's formula [1,2] up to order a $m$. The term of order $0(\mathrm{am})^{2}$ appears to be new.

\section{REFERENCES}

1. KANWAL, R. P. Dynamical displacements in an infinite elastic space and matched asymptotic expansions, J. Math. and Phys. 47 (1965), pp. 273-283.

2. KANWAL, R. P. Integral equation formulation of classical elasticity, Quart. App1. Math. 27 (1969), pp. 57-65.

3. KANWAL, R. P. and D. L. Sharma. Singularity Methods of elastostatics, J. Elasticity 6 (1976), pp. 405-418.

4. DATTA, S. and R. P. Kanwa1. Rectilinear oscillations of a rigid spheroid in an elastic medium, Quart. Appl. Math. 37 (1979), pp. 86-91.

5. DATTA, S. and R. P. Kanwal. Slow torsional oscillations of a spheroidal inclusion in an elastic medium, Utilitas Math. 16 (1979), pp. 111-122.

6. ALAWNEH, A. D. and R. P. Kanwal. Singularity methods in mathematical physics, SIAM REVIEW 10 (1977), pp. 437-471.

7. ALAWNEH, A. D. and N. T. Swagfeh. Singularity method for longitudinal oscillations of axially symmetric bodies in Stokes flow, DIRASAT, The Jordan University, to appear.

8. KANWAL, R. P. Approximations and short cuts based on generalized functions, Proceedings Second Int. Symp. Num. Analy. in App1. Sci 4 Engng, University Press of Virginia, (1980), pp. 531-542. 


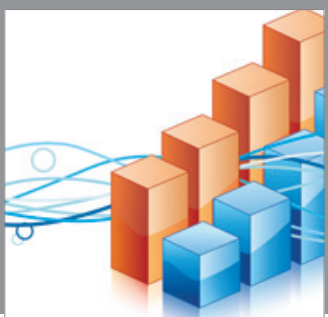

Advances in

Operations Research

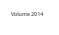

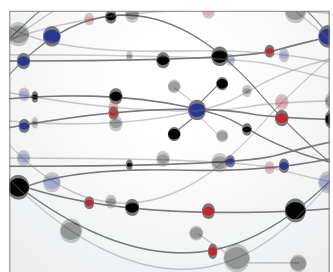

\section{The Scientific} World Journal
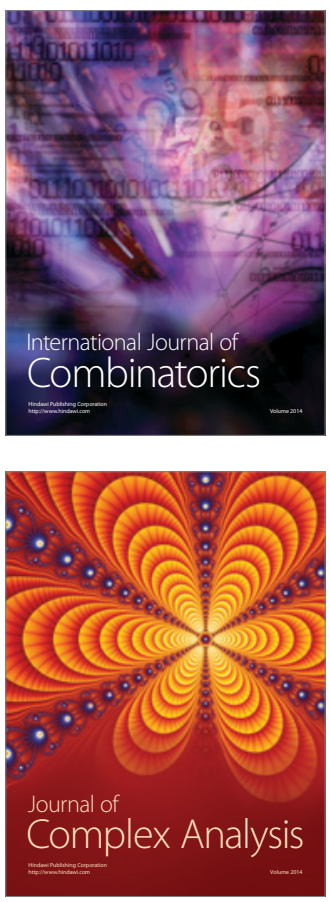

International Journal of

Mathematics and

Mathematical

Sciences
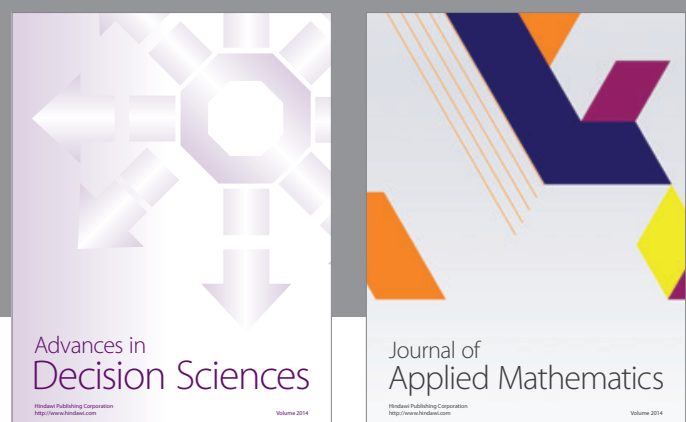

Journal of

Applied Mathematics
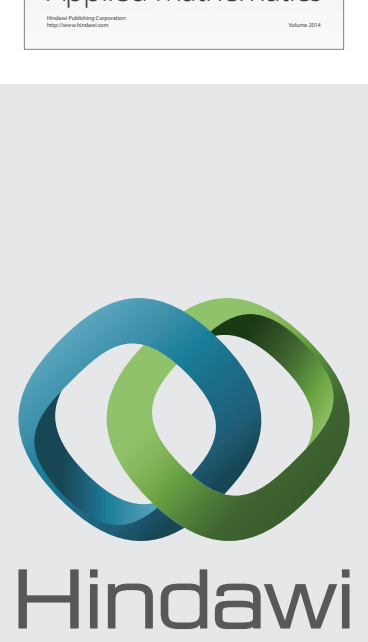

Submit your manuscripts at http://www.hindawi.com
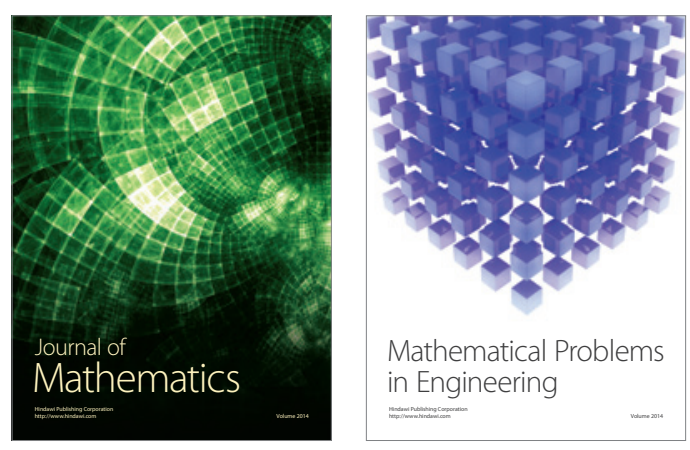

Mathematical Problems in Engineering
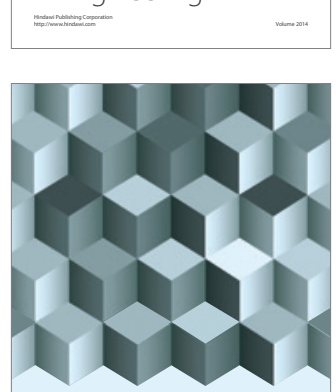

Journal of

Function Spaces
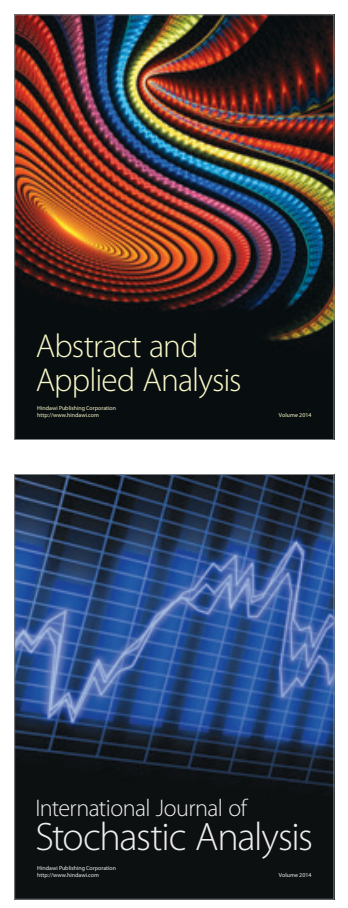

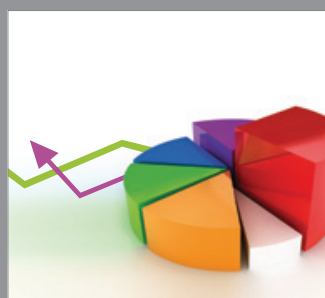

ournal of

Probability and Statistics

Promensencen
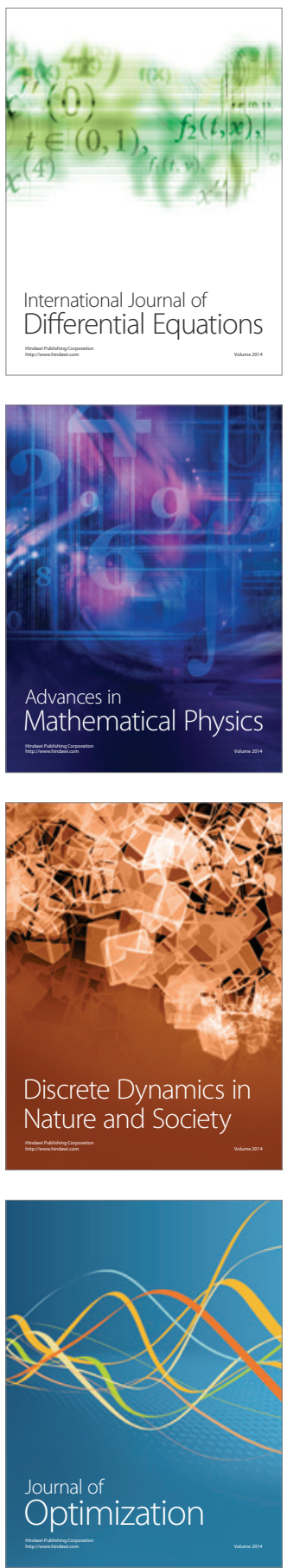